\title{
Contact surgery and transverse invariants
}

\author{
PAOLO Lisca \\ András I. STIPSICZ \\ Dipartimento di Matematica "L. Tonelli" \\ Largo Bruno Pontecorvo, 5 \\ Università di Pisa \\ 56127 Pisa, ITALY \\ Rényi Institute of Mathematics \\ Hungarian Academy of Sciences \\ H-1053 Budapest \\ Reáltanoda utca 13-15, Hungary \\ Email: lisca@dm.unipi.it stipsicz@math-inst.hu
}

\begin{abstract}
The purpose of this paper is two-fold: (1) to derive new existence results for tight contact structures on closed 3-manifolds presented by integral surgery along knots in $S^{3}$, and (2) to introduce a new invariant for transverse knots in contact 3-manifolds. Regarding (1), we extend our previous existence results from surgeries along knots of genus $g$ and maximal Thurston-Bennequin number $2 g-1$ to surgeries along knots of genus $g$ and maximal self-linking number $2 g-1$.
\end{abstract}

AMS Classification 57R17; 57R57

Keywords Tight contact structures, contact surgery, Ozsváth-Szabó invariants

\section{Introduction}

One of the motivating questions of 3-dimensional contact topology is to characterize those (closed, oriented) 3-manifolds which admit (positive, cooriented) tight contact structures. This question was answered recently for Seifert fibered 3-manifolds [21, but the general case is still wide open. A particular family of 3 -manifolds is given by those which can be presented as surgery along a knot in $S^{3}$. It seems reasonable to expect that the use of the invariant $\widehat{\mathcal{L}}(\kappa)$ for Legendrian knots in $S^{3}$ defined in [22] together with contact geometric constructions might provide a way to find tight examples on many such surgeries. Some justification for such an expectation is provided by a result of Sahamie [31, showing that if the Legendrian invariant $\widehat{\mathcal{L}}(\kappa)$ of a Legendrian knot $\kappa$ vanishes, then the contact Ozsváth-Szabó invariant $c\left(\xi_{1}(\kappa)\right)$ of the result of 
contact $(+1)$-surgery along $\kappa$ is also zero. On the other hand, for a Legendrian knot $\kappa$ in the standard contact $S^{3}$ satisfying $\operatorname{tb}(\kappa)=2 g_{s}(\kappa)-1>0$ (where $g_{s}(\kappa)$ denotes the smooth 4 -ball genus of the knot type of $\kappa$ ) it was shown in [20] that the result of contact $(+1)$-surgery has nonvanishing contact Ozsváth-Szabó invariant, implying in particular tightness for the contact structure. (The nonvanishing of this invariant implies tightness, while a contact structure with vanishing invariant might be either tight or overtwisted.)

In this paper we extend this nonvanishing result to knots with other properties, allowing contact surgeries with higher coefficients. Given a knot type $K \subset S^{3}$, let the maximal self-linking number of $K$ be the largest self-linking number of a transverse representative of $K$ (with respect to the standard contact structure $\left.\xi_{s t}\right)$. Also, denote the Seifert genus of $K$ by $g(K)$.

Theorem 1.1 Let $K \subset S^{3}$ be a knot type with maximal self-linking number equal to $2 g(K)-1$. Then, for $r \geq 2 g(K)$ the 3 -manifold $S_{r}^{3}(K)$ carries tight contact structures.

Examples of knots satisfying the assumptions of the above theorem are provided by strongly quasi-positive, fibered knots in $S^{3}$. In particular, iterated torus knots $K\left(\left(p_{1}, q_{1}\right), \ldots,\left(p_{k}, q_{k}\right)\right)$ with all $p_{i}, q_{i}>0$ are such examples.

According to [10] the $(2,3)$-cable $K_{2,3}$ of the $(2,3)$ torus knot $T_{2,3}$ provides an example of a knot for which Theorem 1.1 applies while the previous result from [20] does not: the maximal self-linking of $K_{2,3}$ is equal to 7 (which is equal to $2 g\left(K_{2,3}\right)-1$ ) while the maximal Thurston-Bennequin number of $K_{2,3}$ is 6 . By taking the connected sum of $n$ copies of this knot, the difference between the maximal self-linking and the maximal Thurston-Bennequin number can be made, in fact, arbitrarily large. Related, prime knot examples for the same phenomenon are provided by $(p, q)$-cables $(q>p \geq 1) K_{p, q}$ of the $(2,3)$ torus knot $T_{2,3}$ : according to [11] the maximal self-linking number of $K_{p, q}$ (which again coincides with $2 g\left(K_{p, q}\right)-1$ ) is equal to $p q+q-p$, while the maximal Thurston-Bennequin number of $K_{p, q}$ is $p q$.

We found it convenient to organize the surgery theoretic information about a Legendrian (and about a transverse) knot into an invariant which takes its values in Heegaard Floer homology groups (and ultimately in the inverse limit of some of these groups). Although the resulting surgery invariant $\tilde{c}$ shares a number of properties with the Legendrian (and transverse) knot invariants introduced in [22], we found a vanishing result for $\tilde{c}$ (given in Theorem 1.3) 
which is, according to a recent result of Vela-Vick [32, in sharp contrast with the corresponding behaviour of the Legendrian invariant $\widehat{\mathcal{L}}$ of $[22]$.

In order to state our results we need some preliminary notation. Let $Y$ be a closed, oriented 3-manifold and $K \subset Y$ a knot type. Let $\mathcal{F}_{K}$ be the set of framed isotopy classes of framed knots in the (unframed) knot type $K$. We will follow the usual practice of referring to the elements of $\mathcal{F}_{K}$ as to the "framings" of $K$. Recall that for $K$ null-homologous $\mathcal{F}_{K}$ is an affine $\mathbb{Z}$-space, and that even if $K$ is not null-homologous this is still true if $Y$ is not of the form $Y^{\prime} \# S^{1} \times S^{2}$ [3, 18]. For $k \in \mathbb{Z}$ and $f \in \mathcal{F}_{K}$, we shall denote the result of $k$ acting on $f$ by $f+k$. When $\mathcal{F}_{K}$ is an affine $\mathbb{Z}$-space, $\mathcal{F}_{K}$ inherits a natural linear order from $\mathbb{Z}$ : if $f, g \in \mathcal{F}_{K}$ with $f=g+k, k \in \mathbb{Z}$, then $f \geq g$ if and only if $k \geq 0$. We will denote by $Y_{f}(K)$ the 3 -manifold resulting from surgery on $Y$ along $K$ with framing $f$.

Given a contact $3-$ manifold $(Y, \xi)$, a framed Legendrian knot in $(Y, \xi)$ is a pair $(\kappa, f)$, where $\kappa \subset(Y, \xi)$ is a Legendrian knot and $f \in \mathcal{F}_{K}$ is a framing of the topological type $K$ of $\kappa$. A framed transverse knot in $(Y, \xi)$ is a pair $(\tau, f)$, where $\tau \subset(Y, \xi)$ is a transverse knot and $f \in \mathcal{F}_{K}$ is a framing of the topological type $K$ of $\tau$. Denote by $\mathbb{T}(Y, \xi, K, f)$ the set of transverse isotopy classes of framed transverse knots $(\tau, f)$ in $(Y, \xi)$ with $\tau$ in the topological type $K$. Let $\operatorname{Cont}(Y)$ be the set of isomorphism classes of contact structures on $Y$. Fix a transverse knot $\tau \subset(Y, \xi)$ in the knot type $K$. By considering a Legendrian approximation $\kappa$ of $\tau$, and by applying appropriate contact surgery along $\kappa$ (where the exact meaning of 'appropriate' will be clarified in Subsection 3.1), a contact structure $\tilde{I}(\xi, \tau, f)$ can be defined on the 3 -manifold $Y_{f}(K)$.

Theorem 1.2 Let $Y$ be a closed, oriented 3-manifold and $K$ a knot type in $Y$. Suppose that either $K$ is null-homologous or $Y$ is not of the form $Y^{\prime} \# S^{1} \times S^{2}$. Given a contact structure $\xi$ on $Y$ and a framing $f$ on $K \subset Y$, there is a well-defined map

$$
\begin{array}{ccc}
\mathbb{T}(Y, \xi, K, f) & \longrightarrow & \operatorname{Cont}\left(Y_{f}(K)\right) \\
{[(\tau, f)]} & \longmapsto & \tilde{I}(\xi, \tau, f)
\end{array}
$$

In 28] Ozsváth and Szabó associated an element of the Heegaard Floer group $\widehat{H F}(-Y)$ to every contact 3 -manifold $(Y, \xi)$. By fixing an identification between the diffeomorphic 3-manifolds $Y_{f}(\tau)$ and $Y_{f}(K)$ we get a family of Heegaard Floer elements

$$
\tilde{c}(\xi, \tau, f):=c(\tilde{I}(\xi, \tau, f)) \in \widehat{H F}\left(-Y_{f}(K)\right), \quad f \in \mathcal{F}_{K}
$$


for every transverse knot $\tau \subset(Y, \xi)$ representing the knot type $K$. (In this paper we always consider Heegaard Floer homology with $\mathbb{Z} / 2 \mathbb{Z}$ coefficients). The elements themselves might depend on the chosen identification of $Y_{f}(\tau)$ with $Y_{f}(K)$; their vanishing/nonvanishing properties, on the other hand, are independent of this choice. Since in the following we will exclusively focus on vanishing/nonvanishing questions, we shall not mention the above identification again.

The invariant $\tilde{c}$ is non-trivial. In fact, in Example 3.7 we show, using the main result of [20], that if $\tau$ is the link of an isolated curve singularity in the standard contact 3 -sphere $\left(S^{3}, \xi_{\text {st }}\right)$ then $\tilde{c}\left(\xi_{\text {st }}, \tau, f_{S}+2 g_{s}(K)\right) \neq 0$, where $f_{S}$ is the framing defined by a Seifert surface of $K$, and $g_{s}(K)$ is the slice genus of $K$. In Section 3 we also show (Corollary 3.9) that if $\tilde{c}(\xi, \tau, f) \neq 0$ then $\tilde{c}(\xi, \tau, g) \neq 0$ for every $g \geq f$.

By using appropriate cobordisms and maps induced by them, an inverse limit $H(Y, K)$ of Heegaard Floer groups of results of surgeries of $Y$ along $K$ can be defined, and we show that the family $(\tilde{c}(\xi, \tau, f))_{f \in \mathcal{F}_{K}}$ defines a single element $\tilde{c}(\xi, \tau)$ in this limit (see Proposition 3.8). Notice that $\tilde{c}$ is defined for a transverse knot $\tau$ through its Legendrian approximations, a feature similar to the definition of the transverse invariant $\hat{\mathcal{T}}$ of 22 (resting on the corresponding Legendrian invariant $\widehat{\mathcal{L}})$. For $\tilde{c}$, however, we have the following vanishing result which shows, in particular, that $\tilde{c}(\xi, \tau)$ behaves quite differently from the transverse invariant $\widehat{\mathcal{T}}$ of 22 .

Theorem 1.3 Let $\Sigma$ be an oriented surface with boundary and $\phi: \Sigma \rightarrow \Sigma$ an orientation-preserving diffeomorphism which restricts to the identity on a collar around $\partial \Sigma$. Let $\left(Y, \xi_{(\Sigma, \phi)}\right)$ be the contact 3-manifold compatible with the open book decomposition induced by $(\Sigma, \phi)$. Suppose that $b_{1}(Y)=0$ and $c\left(\xi_{(\Sigma, \phi)}\right)=0$, and let $\tau \subset\left(Y, \xi_{(\Sigma, \phi)}\right)$ be a component of the boundary of $\Sigma$ viewed as the binding of the open book. Then, $\tilde{c}\left(\xi_{(\Sigma, \phi)}, \tau\right)=0$.

Theorem 1.3 should be contrasted with the main result of [32], which says that the transverse invariant $\widehat{\mathcal{T}}$ of 22 ] is nonvanishing for a binding of an open book. (See also [13] for the case of disconnected bindings.)

The following nonvanishing result provides the desired construction of tight contact structures on certain surgered 3-manifolds, and will serve as the main ingredient in the proof of Theorem 1.1 .

Theorem 1.4 Suppose that the open book decomposition induced by $(\Sigma, \phi)$ is compatible with the standard contact structure $\xi_{\text {st }}$ on $S^{3}$. Let $\tau \subset S^{3}$ be a 
binding component of the open book decomposition having knot type $K$ and self-linking number $\operatorname{sl}(\tau)=2 g(K)-1$, where $g(K)$ is the Seifert genus of $K$. Then, $\tilde{c}\left(\xi_{s t}, \tau\right) \neq 0$. In fact, if $f_{S}$ denotes the Seifert framing of $\tau$, the Heegaard Floer homology element $\tilde{c}\left(\xi_{s t}, \tau, f\right)$ is nonzero for each $f \geq f_{S}+2 g(K)$.

The paper is organized as follows. In Section 2 we establish the properties of contact surgeries that we use to define the transverse invariants. In Section 3 we define the invariants, thus establishing Theorem 1.2, and we prove their basic properties. In Section 4 we prove the vanishing Theorem 1.3 while in Section 5 we give the proofs of the nonvanishing result given by Theorem 1.4 and ultimately we prove Theorem 1.1 .

Acknowledgements: We would like to thank John Etnyre and Matt Hedden for stimulating discussions, and the anonymous referee for useful suggestions which helped to improve the presentation. Part of this work was carried out while the authors visited the Mathematical Sciences Research Institute, Berkeley, as participants of the 'Homology theories for knots and links' special semester. The present work is part of the authors' activities within CAST, a Research Network Program of the European Science Foundation. PL was partially supported by PRIN 2007, MIUR. AS was partially supported by OTKA Grant NK81203 and by the Lendület program.

\section{Contact surgeries and stabilizations}

In this section we establish the properties of certain contact surgeries which will allow us to define the invariants and study their basic properties.

Let $K \subset Y$ be a knot type in the closed 3-manifold $Y$. Let $\xi$ be a contact structure on $Y$ and $\kappa \subset(Y, \xi)$ a Legendrian knot belonging to $K$. Recall that, given a non-zero rational number $r \in \mathbb{Q}$, one can perform contact $r$-surgery along $\kappa$ to obtain a new contact 3 -manifold $\left(Y^{\prime}, \xi^{\prime}\right)$ [4]. When $r= \pm 1$ the contact structure $\xi^{\prime}$ is uniquely determined, therefore in this case we can safely use the notation $\xi_{ \pm 1}(\kappa)$ for $\xi^{\prime}$. In general, there are several possible choices for $\xi^{\prime}$. According to [4, Proposition 7], for $\frac{p}{q}>1$ every contact $\frac{p}{q}$-surgery on $\kappa$ is equivalent to a contact $(+1)$-surgery on $\kappa$ followed by a contact $\frac{p}{q-p}$-surgery on a Legendrian pushoff copy of $\kappa$. Moreover, by [4, Proposition 3] (see [5] as well) every contact $r$-surgery along $\kappa \subset(Y, \xi)$ with $r<0$ is equivalent to a Legendrian (i.e. $(-1)-$ ) surgery along a Legendrian link $\mathbb{L}=\cup_{i=0}^{m} L_{i}$ belonging to a set determined via a simple algorithm by the Legendrian knot $\kappa$ and the 
contact surgery coefficient $r$. The algorithm to obtain the set of $\mathbb{L}$ 's is the following. Let

$$
\left[a_{0}, \ldots, a_{m}\right]:=a_{0}-\frac{1}{a_{1}-\frac{1}{\ddots \cdot-\frac{1}{a_{m}}}}, \quad a_{0}, \ldots a_{m} \geq 2,
$$

be the continued fraction expansion of $1-r$. To obtain $L_{0}$, stabilize $a_{0}-2$ times a Legendrian push-off of $\kappa$ in every possible way. Then, stabilize $a_{1}-2$ times a Legendrian push-off of $L_{0}$ in every possible way. Repeat the above scheme for each of the remaining pivots of the continued fraction expansion.

We are interested in contact $n$-surgeries, where $n$ is a positive integer. In this case, since

$$
1-\frac{n}{1-n}=\frac{2 n-1}{n-1}=[3, \overbrace{2, \ldots, 2}^{n-2}],
$$

there are only two choices for the stabilizations of $\kappa$, because the choice of the first one determines all the others. An orientation of $\kappa$ allows one to specify unambiguously such a choice, because it specifies the negative stabilization $\kappa_{-}$ and the positive stabilization $\kappa_{+}$of $\kappa$. In a standard neighborhood $\mathbb{R} / \mathbb{Z} \times \mathbb{R}^{2}$ of $\kappa$ with coordinates $(\theta, x, y)$ the contact structure is given by $\xi=\operatorname{ker}(d x+y d \theta)$, and the $(\theta, x)$-projections of $\kappa, \kappa_{-}$and $\kappa_{+}$are illustrated in Figure 1. From
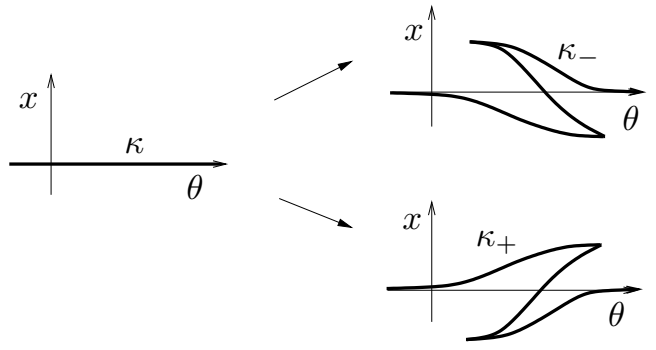

Figure 1: Negative and positive Legendrian stabilizations

now on we shall assume that every Legendrian knot $\kappa$ is oriented.

Definition 2.1 We denote by $\xi_{n}^{-}(\kappa)$ (respectively $\xi_{n}^{+}(\kappa)$ ) the contact structure corresponding to the choice of the negative stabilization $\kappa_{-}$(respectively the positive stabilization $\kappa_{+}$) of the oriented Legendrian knot $\kappa$ (see Figure 1).

Observe that $\kappa_{+}$and $\kappa_{-}$inherit an orientation from $\kappa$ in a natural way. We shall always assume that $\kappa_{+}$and $\kappa_{-}$are given the orientation induced by $\kappa$. 
Lemma 2.2 Let $\kappa \subset(Y, \xi)$ be an oriented Legendrian knot. Then, for each $n>0$ we have

$$
\xi_{n}^{+}(\kappa)=\xi_{n}^{-}(-\kappa)
$$

Proof The statement follows from the definition of contact surgery together with the easily checked fact that $(-\kappa)_{-}=-\left(\kappa_{+}\right)$for every oriented Legendrian knot $\kappa$.

We want to study the contact structure $\xi_{n}^{-}(\kappa)$ when $\kappa$ is a stabilization. The following lemma was proved in greater generality in [23] using the main result of [17]. Here we give a simple and constructive proof.

Lemma 2.3 Let $\kappa \subset(Y, \xi)$ be an oriented Legendrian knot. Then, $\xi_{n}^{-}\left(\kappa_{+}\right)$is an overtwisted contact structure for each $n>0$.

Proof Ozbagci [23, Proposition 13] shows that for $r>0$ any contact $r$-surgery on a positive stabilization in which the Legendrian pushoffs are all negative stabilizations is overtwisted, by constructing a non-right veering compatible open book and appealing to the results of [17].

On the other hand, the lemma can be easily checked directly as follows. The left-hand side of Figure 2 illustrates the contact surgery yielding $\xi_{n}^{-}\left(\kappa_{+}\right)$in a standard neighborhood of $\kappa$. The right-hand side of the picture shows how

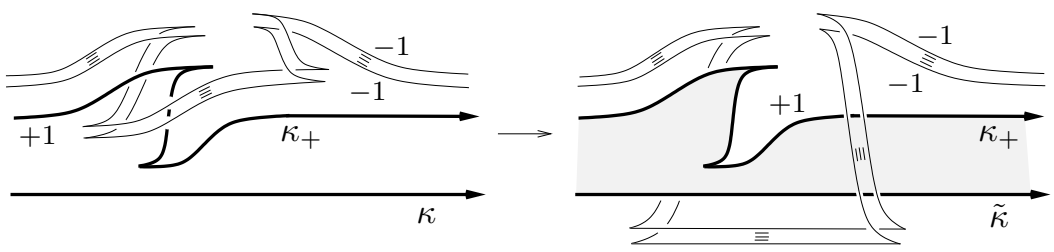

Figure 2: The overtwisted disk in $\xi_{n}^{-}\left(\kappa_{+}\right)$. Notice that the knots labeled by $\kappa$ on the left and $\tilde{\kappa}$ on the right are not necessarily isotopic in the surgered manifold. Nevertheless, $\kappa_{+}$and $\tilde{\kappa}$ provide the shaded annulus, which then caps off to an overtwisted disk with boundary equal to $\tilde{\kappa}$.

the $n-1$ push-offs of $\left(\kappa_{+}\right)_{-}$can be Legendrian isotoped until one can see the shaded overtwisted disk.

The following proposition gives the key property of $\xi_{n}^{-}(\kappa)$ which yields transverse invariants. 
Proposition 2.4 Let $\kappa_{-}$denote the negative Legendrian stabilization of the oriented Legendrian knot $\kappa \subset(Y, \xi)$. Then, for each $n>0$ the contact structures $\xi_{n+1}^{-}\left(\kappa_{-}\right)$and $\xi_{n}^{-}(\kappa)$ are isomorphic.

Before proving the proposition we recall the lantern relation. Let $A$ be a surface with boundary homeomorphic to a twice punctured annulus. If we denote by $\delta_{i}$ the positive Dehn twist along a curve parallel to the $i$-th boundary component of $A$, and by $\delta_{i j}$ the positive Dehn twist along a curve encircling the boundary components $i$ and $j$, the lantern relation reads $\delta_{1} \delta_{2} \delta_{3} \delta_{4}=\delta_{12} \delta_{13} \delta_{23}$. In the proof of Proposition 2.4 we are going to use the equivalent relation $\delta_{12}^{-1} \delta_{1} \delta_{2} \delta_{3}=\delta_{13} \delta_{23} \delta_{4}^{-1}$. Figure 3 provides a graphical representation of this relation. In fact, whenever the twice punctured annulus embeds into a surface, the Dehn twists corresponding to the images of the curves on the diagram satisfy the lantern relation.
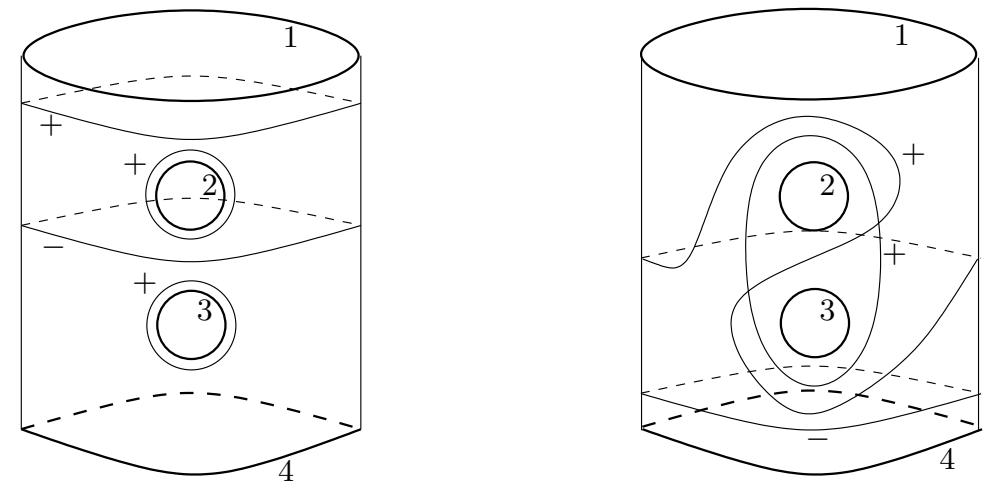

Figure 3: The relation $\delta_{12}^{-1} \delta_{1} \delta_{2} \delta_{3}=\delta_{13} \delta_{23} \delta_{4}^{-1}$. Signs on the curves indicate whether right-handed $(+)$ and left-handed $(-)$ Dehn twists are to be performed.

Proof of Proposition 2.4 Consider an open book for $\xi$ with a page which contains $\kappa$ and such that the page framing induced on $\kappa$ is equal to the contact framing of $\kappa$. After two Giroux stabilizations we can accomodate $\kappa, \kappa_{-}$and $\left(\kappa_{-}\right)_{-}$on the same page of the resulting open book, still with equal page and contact framings (see eg [7]). After performing a negative Dehn twist along $\kappa_{-}$ and positive Dehn twists along $n$ parallel copies of $\left(\kappa_{-}\right)_{-}$we obtain an open book for $\xi_{n+1}^{-}\left(\kappa_{-}\right)$, as illustrated in Figure 4a. In Figure 4b we see what happens to the open book for $\xi_{n+1}^{-}\left(\kappa_{-}\right)$when we apply the relation of Figure 3 inside the twice punctured annulus visible in the picture. The dashed arc of Figure 4b shows that the open book can be Giroux destabilized, yielding Figure 4c, which is an open book for $\xi_{n}^{-}(\kappa)$. 


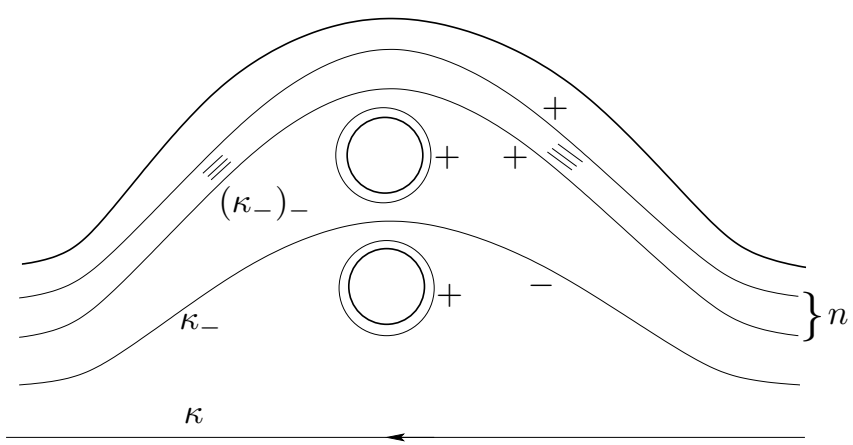

(a) Open book for $\xi_{n+1}^{-}\left(\kappa_{-}\right)$

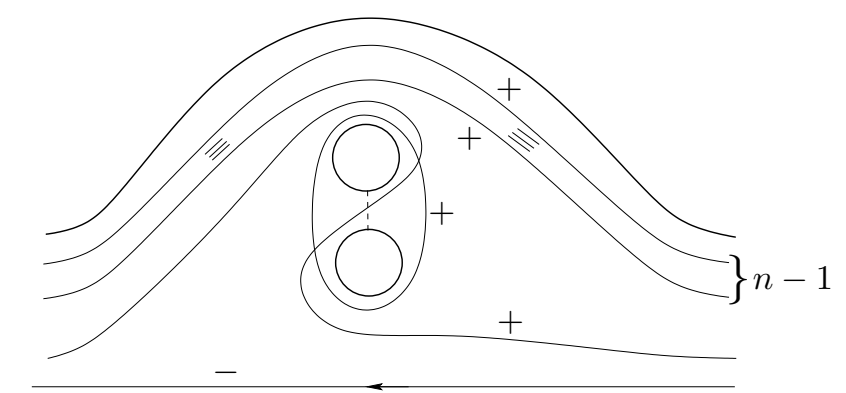

(b) Open book for $\xi_{n+1}^{-}\left(\kappa_{-}\right)$after applying the relation

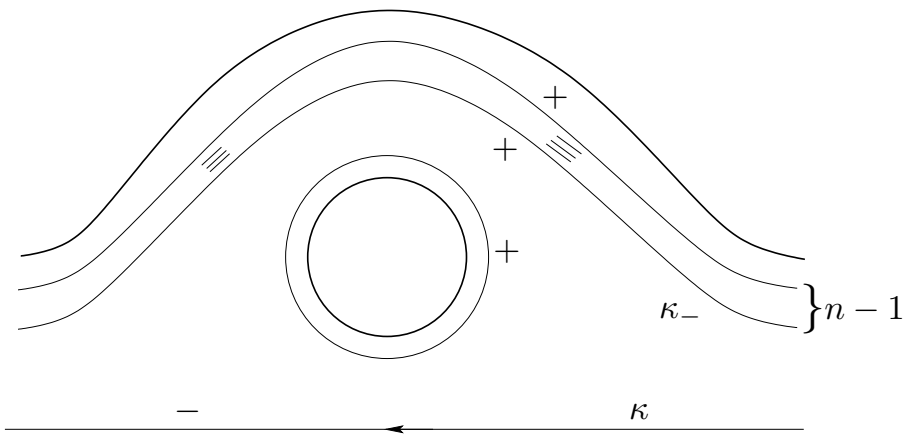

(c) Open book for $\xi_{n}^{-}(\kappa)$

Figure 4: Isomorphism between $\xi_{n+1}^{-}\left(\kappa_{-}\right)$and $\xi_{n}^{-}(\kappa)$

The following corollary can be viewed as a generalization of [8, Theorem 1].

Corollary 2.5 Let $\kappa_{1}, \kappa_{2} \subset(Y, \xi)$ be two Legendrian knots. If after negatively stabilizing the same number of times $\kappa_{1}$ and $\kappa_{2}$ become Legendrian isotopic, then $\xi_{n}^{-}\left(\kappa_{1}\right)$ is isomorphic to $\xi_{n}^{-}\left(\kappa_{2}\right)$ for each $n>0$. 
Proof Suppose that $\kappa_{1}^{\prime}$ and $\kappa_{2}^{\prime}$ are Legendrian isotopic Legendrian knots obtained by negatively stabilizing $\kappa_{1}$ and $\kappa_{2} m$ times. Then, for each $n>0$, the contact structure $\xi_{n+m}^{-}\left(\kappa_{1}^{\prime}\right)$ is isotopic to $\xi_{n+m}^{-}\left(\kappa_{2}^{\prime}\right)$. Applying Proposition 2.4 $m$ times we conclude that $\xi_{n+m}^{-}\left(\kappa_{1}^{\prime}\right)$ is isomorphic to $\xi_{n}^{-}\left(\kappa_{1}\right)$ and $\xi_{n+m}^{-}\left(\kappa_{2}^{\prime}\right)$ is isomorphic to $\xi_{n}^{-}\left(\kappa_{2}\right)$. Therefore $\xi_{n}^{-}\left(\kappa_{1}\right)$ and $\xi_{n}^{-}\left(\kappa_{2}\right)$ are isomorphic for each $n>0$.

Lemma 2.3 and Proposition 2.4 admit slight refinements and alternative proofs, which potentially apply to more general situations (see Remark 2.7 below). We provide the alternative proofs in the following proposition, which is not used in the rest of the paper.

Proposition 2.6 Let $\kappa_{-}$, respectively $\kappa_{+}$, denote the negative, respectively positive, Legendrian stabilization of the oriented Legendrian knot $\kappa \subset(Y, \xi)$. Then, for each $n>0$ we have:

(1) $\xi_{n+1}^{-}\left(\kappa_{-}\right)$is isotopic to $\xi_{n}^{-}(\kappa)$;

(2) $\xi_{n}^{-}\left(\kappa_{+}\right)$is overtwisted.

Proof This simple proposition can be deduced using the foundational results of Ko Honda from [16]. We refer the reader to [16] for the necessary background in what follows. Let us quickly go over the details of the contact surgery construction. The contact framing together with the orientation on $\kappa$ determine an oriented basis $\mu, \lambda$ of the first integral homology group of the boundary of a standard neighborhood $\nu(\kappa)$ of $\kappa$. The basis determines identifications

$$
\partial(\nu(\kappa)) \cong \mathbb{R}^{2} / \mathbb{Z}^{2}, \quad-\partial\left(S^{3} \backslash \nu(\kappa)\right) \cong \mathbb{R}^{2} / \mathbb{Z}^{2} .
$$

The surgery is determined by a gluing prescribed, with repect to the above identifications, by the matrix

$$
A=\left(\begin{array}{cc}
n & -1 \\
1 & 0
\end{array}\right) .
$$

The pull-back of the dividing set is determined by

$$
A^{-1}\left(\begin{array}{l}
0 \\
1
\end{array}\right)=\left(\begin{array}{l}
1 \\
n
\end{array}\right)
$$

so it has slope $n$ on $\partial(\nu(\kappa))$. Applying a diffeomorphism of the solid torus $\nu(\kappa)$ this slope can be changed to $n /(1+n h)$ for any $h \in \mathbb{Z}$. Therefore we can normalized it to lie between -1 and $-\infty$, obtaining slope $-n /(n-1)$. By [16] there are exactly two choices of tight contact structures on the solid torus with 
this boundary slope, corresponding to the two possibile choices (positive or negative) of a basic slice with boundary slopes -1 and $-n /(n-1)$. With our conventions, choosing the negative basic slice gives rise to the contact structure $\xi_{n}^{-}$. The knots $\kappa_{+}$and $\kappa_{-}$can both be realized inside the neighborhood $\nu(\kappa)$. If $\nu\left(\kappa_{ \pm}\right) \subset \nu(\kappa)$ is a standard neighborhood of $\kappa_{ \pm}$, the (closure of the) difference $\nu(\kappa) \backslash \nu\left(\kappa_{ \pm}\right)$is a basic slice, which is positive for $\kappa_{+}$and negative for $\kappa_{-}$[16]. Moreover, its boundary slopes with respect to the basis $\mu, \lambda$ are -1 on $\partial \nu\left(\kappa_{ \pm}\right)$ and $\infty$ on $\partial \nu(\kappa)$.

For each $n \geq 0$, we can perform contact $(n+1)$-surgery along $\kappa_{ \pm}$viewed as a Legendrian knot inside $\nu(\kappa)$, obtaining another contact solid torus $\mathbb{T}$ with convex boundary in standard form. $H_{1}\left(\partial \nu\left(\kappa_{-}\right) ; \mathbb{Z}\right)$ has a basis $\mu^{\prime}, \lambda^{\prime}$ such that, with the obvious identifications, $\mu^{\prime}=\mu$ and $\lambda^{\prime}=\lambda-\mu$. Thus, since $\lambda=\mu^{\prime}+\lambda^{\prime}$, the identity

$$
\left(\begin{array}{cc}
n+1 & -1 \\
1 & 0
\end{array}\right)^{-1}\left(\begin{array}{ll}
0 & 1 \\
1 & 1
\end{array}\right)=\left(\begin{array}{cc}
1 & 1 \\
n+1 & n
\end{array}\right)
$$

implies that, up to applying a diffeomorphism of $\mathbb{T}$, the slopes of $\partial \nu(\kappa)$ and $\partial \nu\left(\kappa_{ \pm}\right)$can be assumed to be, respectively, $-n /(n-1)$ and $-(n+1) / n$. This shows that $\mathbb{T}$ can be decomposed as

$$
\mathbb{T}=N \cup B,
$$

where $N$ is standard neighborhood of a Legendrian curve with slope -1 , and $B \cong T^{2} \times[0,1]$ has boundary slopes -1 and $-n /(n-1)$ and can be written as a union of two basic slices $B=B_{1} \cup B_{2}$, where $B_{1}$ has boundary slopes $(-1,-(n+1) / n)$ and $B_{2}=\nu(\kappa) \backslash \nu\left(\kappa_{ \pm}\right)$, with the boundary slopes given above. Since $B_{2}$ is a basic slice, $B$ is a basic slice (i.e. it is tight) if and only if $B_{1}$ and $B_{2}$ have the same sign as basic classes [16]. By definition, $\xi_{n+1}^{-}\left(\kappa_{ \pm}\right)$is the contact structure obtained by taking $B_{1}$ to be a negative basic slice. Since $B_{2}$ is positive for $\kappa_{+}$and negative for $\kappa_{-}$, the analysis above proves simultaneously (1) and (2) of the statement.

Remark 2.7 While the proof of Proposition 2.4 only holds, as written, for closed contact 3-manifolds, both the statement and the proof of Proposition 2.6 can stay the same even if $(Y, \xi)$ is open or has non-empty boundary. This allows one, at least in principle, to apply the approach of this paper in situations which are more general than the ones considered here. We hope to return to this issue in a future paper.

Remark 2.8 Let $\xi_{n}^{+}(\kappa)$ be the contact structure corresponding to the choice of the positive stabilization $\kappa_{+}$of $\kappa$. Then, an argument analogous to that of the 
proof of Proposition 2.6 shows that $\xi_{n+1}^{+}\left(\kappa_{+}\right)$is isotopic to $\xi_{n}^{+}(\kappa)$ and $\xi_{n+1}^{+}\left(\kappa_{-}\right)$ is overtwisted. Of course, this also follows from the fact that $(-\kappa)_{-}=-\kappa_{+}$ and Proposition 2.6. In fact,

$$
\xi_{n+1}^{+}\left(\kappa_{+}\right)=\xi_{n+1}^{-}\left(-\kappa_{+}\right)=\xi_{n+1}^{-}\left((-\kappa)_{-}\right)=\xi_{n}^{-}(-\kappa)=\xi_{n}^{+}(\kappa)
$$

and

$$
\xi_{n+1}^{+}\left(\kappa_{-}\right)=\xi_{n+1}^{-}\left(-\kappa_{-}\right)=\xi_{n+1}^{-}\left(\kappa_{+}\right)
$$

\section{The invariants: definition and basic properties}

In this section we define the invariants, we prove some of their properties and present some examples.

\subsection{Definition of the geometric invariant $I(\xi, \kappa, f)$}

Let $K$ be the knot type of a Legendrian knot $\kappa \subset(Y, \xi)$, and let $t:=\operatorname{tb}(\kappa) \in \mathcal{F}_{K}$ be the Thurston-Bennequin invariant of $\kappa$, i.e. the contact framing of $\kappa$. Suppose that either $K$ is null-homologous or $Y$ has no $S^{1} \times S^{2}$-summand. For each positive integer $n$, the contact structure $\xi_{n}^{-}(\kappa)$ lives on the closed 3-manifold $Y_{t+n}(K)$ obtained by performing topological surgery along $K$ corresponding to the framing $t+n$.

Let $(\kappa, f)$ be a framed, oriented Legendrian knot in the contact 3-manifold $(Y, \xi)$, and let $\kappa^{\prime} \subset(Y, \xi)$ be a Legendrian knot obtained by negatively stabilizing $\kappa$ sufficientely many times, so that $\operatorname{tb}\left(\kappa^{\prime}\right)<f$. In view of Proposition 2.4, the isomorphism class of the contact structure $\xi_{f-\mathrm{tb}\left(\kappa^{\prime}\right)}^{-}\left(\kappa^{\prime}\right)$ on $Y_{f}(K)$ does not depend on the choice of $\kappa^{\prime}$ as long as $\operatorname{tb}\left(\kappa^{\prime}\right)<f$, therefore we can introduce the following:

Definition 3.1 Assume that either the knot type $K$ is null-homologous or $Y$ is not of the form $Y^{\prime} \# S^{1} \times S^{2}$. Let $(\kappa, f)$ be a framed, oriented Legendrian knot in the contact 3 -manifold $(Y, \xi)$ such that $\kappa$ has topological type $K$. Define $I(\xi, \kappa, f)$ to be the isomorphism class of the contact structure $\xi_{f-\operatorname{tb}\left(k^{\prime}\right)}^{-}\left(\kappa^{\prime}\right)$ on $Y_{f}(K)$, where $\kappa^{\prime} \subset(Y, \xi)$ is any negative stabilization of $\kappa$ such that $\operatorname{tb}\left(\kappa^{\prime}\right)<f$.

Proposition 3.2 Assume that either $K$ is null-homologous or $Y$ is not of the form $Y^{\prime} \# S^{1} \times S^{2}$. Let $(\kappa, f)$ be a framed, oriented Legendrian knot in the contact 3-manifold $(Y, \xi)$ such that $\kappa$ has topological type $K$. Then, $I(\xi, \kappa, f)$ is overtwisted for each $f \leq \operatorname{tb}(\kappa)$. 
Proof By definition, $I(\xi, \kappa, f)$ is the isomorphism class of the contact structure $\xi_{f-\operatorname{tb}\left(\kappa^{\prime}\right)}^{-}\left(\kappa^{\prime}\right)$, where $\kappa^{\prime}$ is any negative stabilization of $\kappa$ such that $\operatorname{tb}\left(\kappa^{\prime}\right)<$ $f$. If $f \leq \operatorname{tb}(\kappa)$ we can choose $\kappa^{\prime}$ so that $\operatorname{tb}\left(\kappa^{\prime}\right)=f-1$. We have $\kappa^{\prime}=\kappa_{-}^{\prime \prime}$ for some oriented Legendrian knot $\kappa^{\prime \prime}$. Then,

$$
\xi_{f-\operatorname{tb}\left(\kappa^{\prime}\right)}^{-}\left(\kappa^{\prime}\right)=\xi_{1}\left(\kappa_{-}^{\prime \prime}\right)=\xi_{1}\left(-\left(-\kappa^{\prime \prime}\right)_{+}\right)
$$

is overtwisted by Lemma 2.3 ,

Recall that transverse knots admit a preferred orientation and can be approximated, uniquely up to negative stabilization, by oriented Legendrian knots $\underline{6}$, 9]. Fix a transverse knot $\tau \subset(Y, \xi)$, and let $\kappa$ be a Legendrian approximation of $\tau$. Then, by [6, 9], up to negative stabilizations the Legendrian knot $\kappa$ only depends on the transverse isotopy class of $\tau$.

It follows immediately from Proposition 2.4 that if $\kappa^{\prime} \subset(Y, \xi)$ is a negative stabilization of the oriented Legendrian knot $\kappa \subset(Y, \xi)$, then for each framing $f$ we have $I\left(\xi, \kappa^{\prime}, f\right)=I(\xi, \kappa, f)$. This observation allows us to give the following:

Definition 3.3 Assume that either $K$ is null-homologous or $Y$ is not of the form $Y^{\prime} \# S^{1} \times S^{2}$. Let $(\tau, f)$ be a framed transverse knot in the contact 3 -manifold $(Y, \xi)$ such that $\tau$ has topological type $K$. Define $\tilde{I}(\xi, \tau, f):=$ $I(\xi, \kappa, f)$, where $\kappa$ is any Legendrian approximation of $\tau$.

Proof of Theorem 1.2 Since the choice of $\kappa$ is unique up to negative stabilization, the repeated application of Proposition 2.4 verifies the result.

\subsection{Heegaard Floer invariants}

We now apply the Heegaard Floer contact invariant defined by Ozsváth and Szabó [28].

Definition 3.4 Let $Y$ be a closed, oriented 3-manifold, $K \subset Y$ a knot type and $f \in \mathcal{F}_{K}$. Assume that either $K$ is null-homologous or $Y$ is not of the form $Y^{\prime} \# S^{1} \times S^{2}$. Given an oriented Legendrian knot $\kappa \subset(Y, \xi)$, define

$$
c(\xi, \kappa, f):=c(I(\xi, \kappa, f)) \in \widehat{H F}\left(-Y_{f}(K)\right),
$$

and given a transverse oriented knot $\tau \subset(Y, \xi)$, define

$$
\tilde{c}(\xi, \tau, f):=c(\tilde{I}(\xi, \tau, f)) \in \widehat{H F}\left(-Y_{f}(K)\right) .
$$


Remarks 3.5 - It follows immediately from the definition, Lemma 2.3 and Proposition 2.4 that, for each $f \in \mathcal{F}_{K}, c\left(\xi, \kappa_{-}, f\right)=c(\xi, \kappa, f)$ and $c\left(\xi, \kappa_{+}, f\right)=0$.

- It follows from Proposition 3.2 that $c(\xi, \kappa, f)=0$ for each $f \leq \operatorname{tb}(\kappa)$.

- If the complement of a Legendrian knot $\kappa$ in $(Y, \xi)$ is overtwisted or has positive Giroux torsion, the same holds for $\xi_{n}^{-}\left(\kappa^{\prime}\right)$ for some stabilization $\kappa^{\prime}$ of $\kappa$. Therefore, it follows from the results of [15, 28, that $c(\xi, \kappa, f)=0$ for each $f \in \mathcal{F}_{K}$.

The following examples show that the invariant $c(\xi, \kappa, f)$ is non-trivial.

Example 3.6 Consider the Legendrian unknot $\kappa \subset\left(S^{3}, \xi_{s t}\right)$ with ThurstonBennequin number -1 . (In this case $\kappa=-\kappa$, so we do not need to specify the orientation). Since the result of contact $(+1)$-surgery is equal to the unique Stein fillable contact structure on $S^{1} \times S^{2}$, we get that $c\left(\xi_{s t}, \kappa, \operatorname{tb}(\kappa)+1\right) \neq 0$ (cf. [19, Lemma 5]).

Example 3.7 Let $\kappa \subset\left(S^{3}, \xi_{s t}\right)$ be an oriented Legendrian knot with knot type $K$ such that

$$
\operatorname{tb}(\kappa)=f_{S}+2 g_{s}(K)-1>0,
$$

where $f_{S}$ is the framing defined by a Seifert surface of $K$, and $g_{s}(K)$ is the slice genus of $K$. Then, by [20, Proof of Theorem 1.1] $c\left(\xi_{s t}, \kappa, \operatorname{tb}(\kappa)+1\right) \neq 0$. As remarked in 20], the knot types containing Legendrian knots which satisfy Condition (3.1) include all non-trivial algebraic knots, i.e. non-trivial knots which are links of isolated curve singularities, as well as negative twist knots.

\subsection{The inverse limit construction}

The invariants $\tilde{c}(\xi, \tau, f)$ can be conveniently organized as a single element in the inverse limit of certain Heegaard Floer homology groups. In the rest of this section we spell out the details of this construction.

Let $Y$ be a closed, oriented $3-$ manifold and $K$ a knot type in $Y$. To each framing $f \in \mathcal{F}_{K}$ one can naturally associate a triangle of 3 -manifolds and cobordisms (cf. [20, pp. 933-935]). Let $Y_{f-1}(K)$ be the 3-manifold resulting from surgery along $K$ with framing $f-1$. The first manifold in the triangle is $Y$, the second is $Y_{f-1}(K)$ and the third one is $Y_{f}(K)$. A cobordism $W_{f}$ from $Y_{f-1}(K)$ to $Y_{f}(K)$ can be given by considering a normal circle $N$ to $K$ in $Y$, equip if with framing $f_{S}-1$, and after the surgery on $K$ with framing $f-1$ 
has been performed, attach a 4-dimensional 2-handle along $N$ with the chosen framing. Simple Kirby calculus shows that $W_{f}$ is indeed a cobordism between $Y_{f-1}(K)$ and $Y_{f}(K)$.

When viewed upside down, $W_{f}$ induces a map

$$
\widehat{F}_{\bar{W}_{f}}: \widehat{H F}\left(-Y_{f}(K)\right) \rightarrow \widehat{H F}\left(-Y_{f-1}(K)\right) \text {. }
$$

Given framings $f \geq g$, define $\varphi_{g, f}$ to be the identity on $\widehat{H F}\left(-Y_{f}(K)\right)$ if $f=g$, and the composition

$$
\widehat{H F}\left(-Y_{f}(K)\right) \stackrel{F_{\bar{W}_{f}}}{\longrightarrow} \widehat{H F}\left(-Y_{f-1}(K)\right) \longrightarrow \cdots \stackrel{F_{\bar{W}_{g+1}}}{\longrightarrow} \widehat{H F}\left(-Y_{g}(K)\right)
$$

if $f>g$. Then, it is easy to check that the set

$$
\left\{\left(\widehat{H F}\left(-Y_{f}(K)\right), \varphi_{g, f}\right)\right\}
$$

is an inverse system of $\mathbb{Z} / 2 \mathbb{Z}$-vector spaces and linear maps over the set $\mathcal{F}_{K}$, so we can form the inverse limit $\mathbb{Z} / 2 \mathbb{Z}$-vector space

$$
H(Y, K):=\lim \widehat{H F}\left(-Y_{f}(K)\right),
$$

which is the subspace

$$
\left\{\left(x_{f}\right) \in \prod_{f \in \mathcal{F}_{K}} \widehat{H F}\left(-Y_{f}(K)\right) \mid x_{g}=\varphi_{g, f}\left(x_{f}\right) \text { for } g \leq f\right\} \subset \prod_{f \in \mathcal{F}_{K}} \widehat{H F}\left(-Y_{f}(K)\right) .
$$

We define $\tilde{c}(\xi, \tau)$ as the vector $(c(\tilde{I}(\xi, \tau, f)))_{f \in \mathcal{F}_{K}}$, which is, a priori an element of $\prod_{f \in \mathcal{F}_{K}} \widehat{H F}\left(-Y_{f}(K)\right)$.

Proposition 3.8 The invariant $\tilde{c}(\xi, \tau)$ is in $H(Y, K)$.

Proof Choose $f \in \mathcal{F}_{K}$ and a negative stabilization $\kappa^{\prime}$ of $\kappa$, with $\operatorname{tb}\left(\kappa^{\prime}\right)<$ $f-1$. By [4], performing contact $(+1)$-surgery on an extra push-off copy of $\kappa_{-}^{\prime}$ in the contact surgery presentation for $\xi_{f-\mathrm{tb}\left(\kappa^{\prime}\right)}^{-}\left(\kappa^{\prime}\right)$ gives $\xi_{f-1-\mathrm{tb}\left(\kappa^{\prime}\right)}^{-}\left(\kappa^{\prime}\right)$. The corresponding 2-handle attachment gives an oriented 4-dimensional cobordism from $Y_{f}(K)$ to $Y_{f-1}(K)$, and it is easy to check that reversing the orientation of that cobordism gives exactly the oriented cobordism $\bar{W}_{f}$. By [28, Theorem 2.3] (see also [20, Theorem 2.2]), we have

$$
\widehat{F}_{\bar{W}_{f}}\left(c\left(\xi_{f-\operatorname{tb}\left(\kappa^{\prime}\right)}^{-}\left(\kappa^{\prime}\right)\right)\right)=c\left(\xi_{f-1-\operatorname{tb}\left(\kappa^{\prime}\right)}^{-}\left(\kappa^{\prime}\right)\right),
$$

i.e.

$$
\varphi_{f-1, f}(c(I(\xi, \kappa, f)))=c(I(\xi, \kappa, f-1)) .
$$


Since this holds for each $f \in \mathcal{F}_{K}$, and for $g \leq f$ we have

$$
\varphi_{g, f}=\varphi_{g+1, g} \circ \cdots \circ \varphi_{f-1, f}
$$

the statement is proved.

Proposition 3.8 immediately gives the following:

Corollary 3.9 If $c(\xi, \kappa, g) \neq 0$ then $c(\xi, \kappa, f) \neq 0$ for every $f \geq g$.

\section{Proof of Theorem 1.3}

Let $\Sigma$ be an oriented surface-with-boundary and $\phi: \Sigma \rightarrow \Sigma$ an orientationpreserving diffeomorphism which restricts to the identity on a collar around $\partial \Sigma$. Let $\left(Y, \xi_{(\Sigma, \phi)}\right)$ be a contact 3-manifold compatible with the open book decomposition induced by $(\Sigma, \phi)$. Let $\tau \subset\left(Y, \xi_{(\Sigma, \phi)}\right)$ be a component of the boundary of $\Sigma$ viewed as the binding of the open book and let $f_{\Sigma}$ be the framing induced on $\tau$ by $\Sigma$.

Proposition 4.1 There exists a Legendrian approximation $\kappa \subset(Y, \xi)$ to $\tau$ such that $\operatorname{tb}(\kappa)=f_{\Sigma}-1$ and, for each $n>0$, the contact structure $\xi_{n}^{-}(\kappa)$ admits a compatible open book with a binding component $\tau^{\prime}$ having the following properties:

- Capping-off $\tau^{\prime}$ gives back the open book $(\Sigma, \phi)$;

- Let $Z$ be the cobordism corresponding to capping-off $\tau^{\prime}$, and let $X_{\kappa, n}$ be the topological cobordism obtained by attaching a 4-dimensional 2handle along $\kappa$ with framing $\operatorname{tb}(\kappa)+n$. Then, $\bar{Z}=-X_{\kappa, n}$, i.e. $Z$ is obtained from $X_{\kappa, n}$ by viewing it upside-down and reversing its orientation.

Proof As shown in [32, Lemma 3.1], any open book decomposition can be Giroux stabilized so that the page of the new open book $\left(\Sigma^{\prime}, \phi^{\prime}\right)$ contains a Legendrian approximation $\kappa$ of $\tau$ as a curve sitting on a page $\Sigma^{\prime}$ and parallel to (a component of) $\partial \Sigma^{\prime}$, with $\operatorname{tb}(\kappa)=f_{\Sigma^{\prime}}=f_{\Sigma}-1$. This is illustrated in Figure 5a-b. As shown in Figure $5 \mathrm{~b}-\mathrm{c}$, after a further Giroux stabilization both $\kappa$ and its negative stabilization $\kappa_{-}$can be Legendrian realized on the page of an open book $\left(\Sigma^{\prime \prime}, \phi^{\prime \prime}\right)$. The knot $\kappa_{-}$is parallel on $\Sigma^{\prime \prime}$ to a boundary component which coincides with $\tau$, as indicated in Figure 5 r. Performing contact $(+n)-$ surgery along $\kappa$ is equivalent to a contact $(+1)$-surgery along $\kappa$ plus contact 


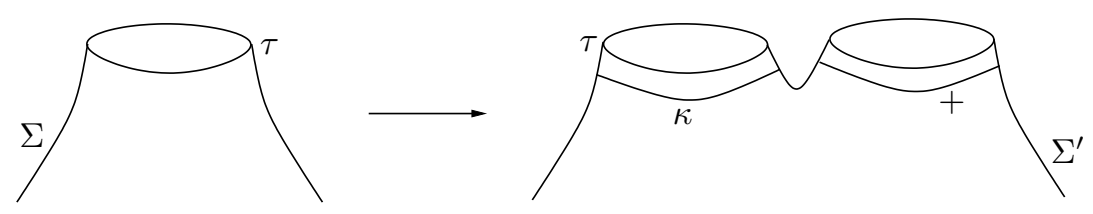

(a)

(b)

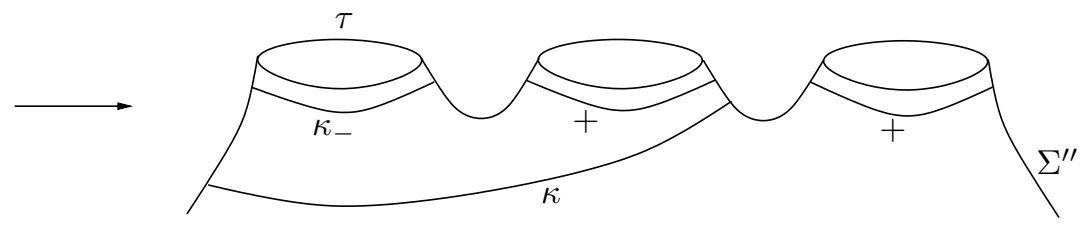

(c)

Figure 5: Legendrian approximation of the binding. Once again, the signs \pm on the curves indicate whether right- or left-handed Dehn twists are to be performed on the given curve.

(-1)-surgeries along $n-1$ parallel copies of $\kappa_{-}$. Therefore, the resulting contact structure is supported by the open book obtained by composing $\phi^{\prime \prime}$ with a negative Dehn twist along the curve corresponding to $\kappa$, as well as positive Dehn twists along $n-1$ parallel copies of the curve corresponding to $\kappa_{-}$. This is illustrated in Figure 6 $a$. As shown in Figure 6 $6-b$, capping-off

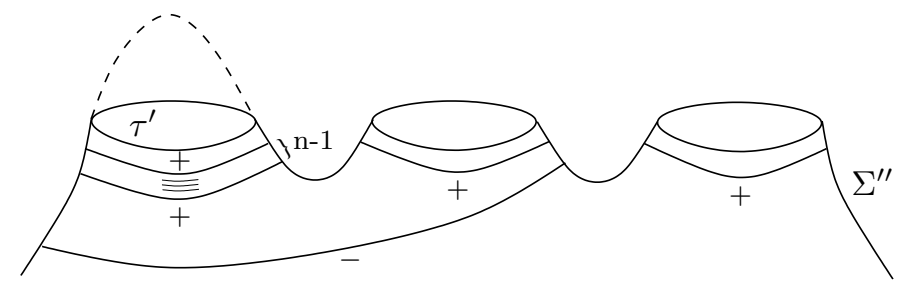

(a)

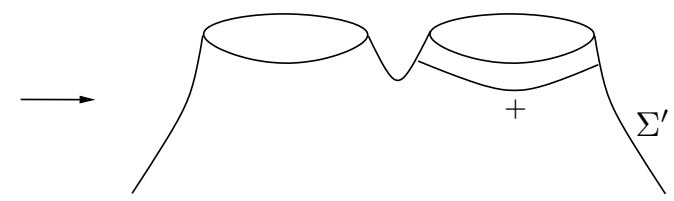

(b)

Figure 6: Contact $n$-surgery and capping-off.

the binding component denoted $\tau^{\prime}$ in the picture yields the open book $\left(\Sigma^{\prime}, \phi^{\prime}\right)$. (Notice the cancellation of the left-handed Dehn twist of Figure 6 a after the 
capping-off.) This proves the first part of the statement.

In order to control what happens at the level of 4-dimensional 2-handles, we represent the surgeries inside a standard Legendrian neighborhood of $\kappa$, as illustrated in Figure 7 $\mathrm{k}$. The framing coefficients appearing in the picture

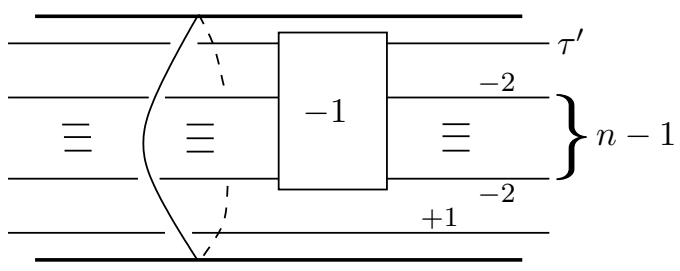

(a)

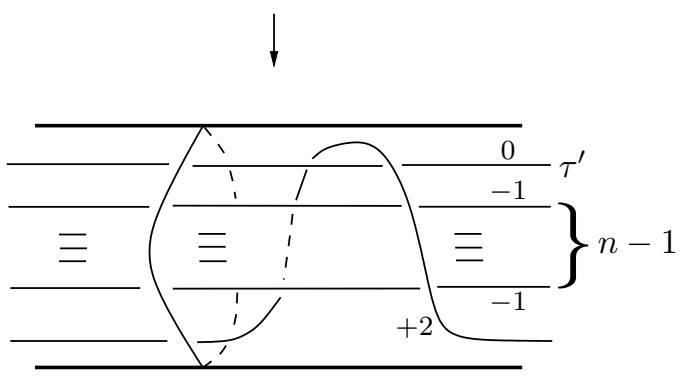

(b)

Figure 7: Contact $(+n)$-surgery along $\kappa$ in a standard neighborhood.

have the following significance. Observe that each curve isotopic to the core of the solid torus has a canonical framing coming from the identification of the solid torus with $S^{1} \times D^{2}$. We have chosen the identification of a neighborhood of $\kappa$ with $S^{1} \times D^{2}$ so that the canonical framing of the core corresponds to the contact framing of $\kappa$. With this convention, the framing induced by $\Sigma^{\prime \prime}$ on $\tau^{\prime}$ would be denoted by " -1 " in Figure 7 $\mathrm{a}$. Figure 7b describes the surgery when we change the solid torus identification by a "right-handed twist". Then, the cobordism $Z$ corresponding to capping-off $\tau^{\prime}$ is obtained by attaching a 4-dimensional 2-handle along $\tau^{\prime}$ with framing 0, as shown in Figure $7 \mathrm{~b}$. After sliding $\tau^{\prime}$ over one of the $(-1)$-framed circles in Figure $7 \mathrm{~b}$, and then repeatedly the $(-1)$-framed circles over each other, and finally blowing up the last two curves, we arrive at Figure $8 \mathrm{a}$. A handle slide, followed by a blow-down gives Figure 8 $\mathrm{b}$, and further blow-downs give Figure 8 $\mathrm{r}$. Applying a "left-handed twist" to the solid torus neighborhood (which just undoes the "right-handed twist" we applied earlier) gives Figure $8 \mathrm{~d}$. This shows that $Z$ can be viewed 


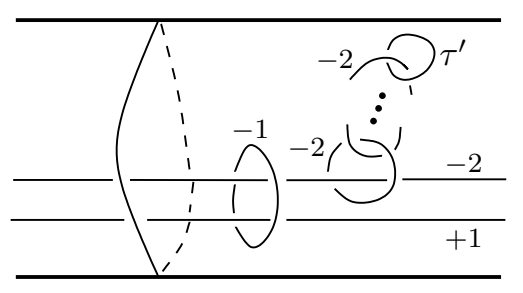

(a)

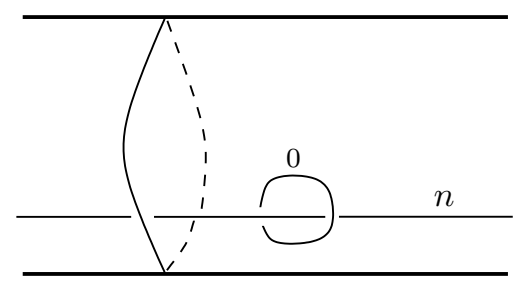

(d)

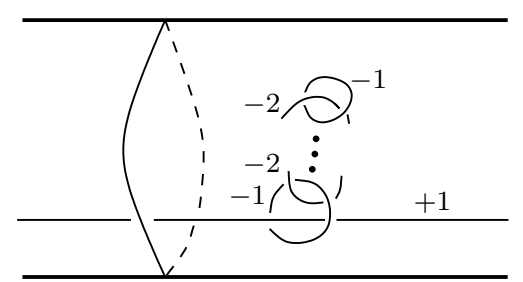

(b)

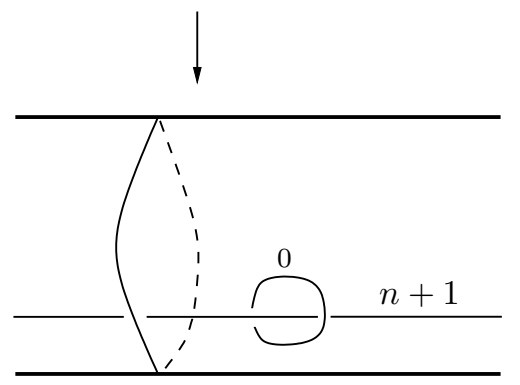

(c)

Figure 8: Kirby moves in a standard neighborhood. In 8 a there are $n-2$ small $(-2)$-framed circles and a further long $(-2)$-framed circle. In $8 \mathrm{~b}$ we have $n-2$ $(-2)$-framed circles.

as the cobordism obtained by attaching a 2 -handle along a meridian to the original curve $\kappa$, with framing 0 with respect to the meridian disk. The picture shows that $Z$ coincides precisely with $-\overline{X_{\kappa, n}}$, where $X_{\kappa, n}$ is the cobordism obtained by attaching a 2 -handle along $\kappa$ with framing $+n$ with respect to the contact framing, the minus sign denotes orientation-reversal and the overline bar means viewing the cobordism "up-side down".

Proof of Theorem 1.3 By Baldwin's theorem [2, Theorem 1.2], for each positive integer $n$ there is a $\operatorname{Spin}^{c}$ structure $\mathbf{s}_{0}$ on the cobordism $\bar{Z}$ such that

$$
F_{\bar{Z}, \mathbf{s}_{0}}(c(\xi))=c\left(\xi_{n}^{-}(\kappa)\right)
$$

This equation then clearly shows that if $c(\xi)=0$ then $\tilde{c}(\xi, \tau, f)=0$ for each $f \geq f_{\Sigma}$, verifying that the element $\tilde{c}(\xi, \tau) \in H(Y, K)$ has only vanishing components, hence $\tilde{c}(\xi, \tau)=0$. 


\section{Proofs of Theorem 1.1 and Theorem 1.4}

Let $Y$ be a closed, oriented rational homology 3 -sphere, and let $\xi$ be a contact structure on $Y$. Let $\kappa \subset(Y, \xi)$ be an oriented Legendrian knot. Suppose that $\kappa=\partial \Sigma$, where $\Sigma \subset Y$ is an embedded oriented surface.

Let $X$ be the oriented 4-dimensional cobordism obtained by attaching a 4 dimensional 2-handle $H$ to $Y$ along $\kappa$. Let $[\Sigma \cup D] \in H_{2}(X ; \mathbb{Z})$ denote the homology class supported by the union of $\Sigma$ and the core $D$ of $H$.

Lemma 5.1 If $\mathbf{s}_{1}$ and $\mathbf{s}_{2}$ are Spin ${ }^{c}$ structures on $X_{\kappa, n}$ having the same restriction to $Y$ and satisfying

$$
\left\langle c_{1}\left(\mathbf{s}_{1}\right),[\Sigma \cup D]\right\rangle=\left\langle c_{1}\left(\mathbf{s}_{2}\right),[\Sigma \cup D]\right\rangle,
$$

then $\mathbf{s}_{1}=\mathbf{s}_{2}$.

Proof The set of $\operatorname{Spin}^{c}$ structures on $X$ which restrict to $Y$ as a fixed $\operatorname{Spin}^{c}$ structure is an affine space on $H^{2}(X, Y)$ (with integral coefficients). Since $Y$ is a rational homology sphere and $X$ is obtained up to homotopy by attaching a 2 -disk to $Y$, by excision we have $H_{2}(X, Y) \cong \mathbb{Z}$ and $H^{2}(X, Y) \cong \mathbb{Z}$. The exact homology sequence of the pair $(X, Y)$ shows that the map $i_{*}: H_{2}(X) \rightarrow$ $H_{2}(X, Y)$ is injective, and therefore $H_{2}(X) \cong \mathbb{Z}$, with generator $[\Sigma \cup D]$. The exact cohomology sequence shows that the restriction map $i^{*}: H^{2}(X, Y) \rightarrow$ $H^{2}(X)$ is injective, and the free part of $H^{2}(X)$ has rank 1 . The evaluation map

$$
H^{2}(X) \rightarrow \operatorname{Hom}\left(H_{2}(X) ; \mathbb{Z}\right) \cong \mathbb{Z}, \quad \beta \mapsto\langle\beta,[\Sigma \cup D]\rangle
$$

is surjective. Therefore, the composition of $i^{*}$ with the evaluation map is injective. If $\mathbf{s}_{1}, \mathbf{s}_{2}$ are two $\operatorname{Spin}^{c}$ structures on $X$ with coinciding restriction to $Y$, then $\mathbf{s}_{1}-\mathbf{s}_{2}=\alpha$, where $\alpha$ belongs to the image of $i^{*}$, and $c_{1}\left(\mathbf{s}_{1}\right)-c\left(\mathbf{s}_{2}\right)=2 \alpha$. Therefore, if the evaluation map takes the same value on $c_{1}\left(\mathbf{s}_{1}\right)$ and $c_{2}\left(\mathbf{s}_{2}\right)$, it follows that $\alpha=0$, hence $\mathbf{s}_{1}=\mathbf{s}_{2}$.

Let tb denote the Thurston-Bennequin number of $\kappa$ with respect to $\Sigma$, and let rot be the rotation number of $\kappa$ with respect to $\Sigma$. Fix $n>0$, and let $X_{\kappa, n}$ be the oriented 4-dimensional cobordism obtained by attaching a 4-dimensional $2-$ handle to $Y$ along $\kappa$ with framing $\mathrm{tb}+n$.

Proposition 5.2 There exists a $\operatorname{Spin}^{c}$ structure $\mathbf{s}$ on $X_{\kappa, n}$ such that:

(1) $\mathbf{s}$ extends the Spin ${ }^{c}$ structures induced on $\partial X_{\kappa, n}$ by $\xi_{s t}$ and $\xi_{n}^{-}(\kappa)$; 
(2) $\frac{1}{4}\left(c_{1}(\mathbf{s})^{2}-3 \sigma\left(X_{\kappa, n}\right)-2 \chi\left(X_{\kappa, n}\right)\right)+1=d_{3}\left(\xi_{n}^{-}(\kappa)\right)-d_{3}\left(\xi_{s t}\right)$;

(3) $\left\langle c_{1}(\mathbf{s}),[\Sigma \cup D]\right\rangle=\operatorname{rot}+n-1$.

Proof We can view Figure 7a (ignoring the knot $\tau$ ) as $S^{3}$ union $n$ 4-dimensional 2 -handles. The sequence of Figures $8 \mathrm{a}, 8 \mathrm{~b}$ and $8 \mathrm{r}$ shows that in fact Figure $7 \mathrm{a}$ represents $\widehat{X}_{\kappa, n}:=X_{\kappa, n} \#(n-1) \overline{\mathbb{C P}}^{2}$. By e.g. [5, Section 3], $\widehat{X}_{\kappa, n} \# \mathbb{C P}^{2}$ carries an almost complex structure $J$ inducing 2 -plane fields homotopic to $\xi_{s t}$ and $\xi_{n}^{-}(\kappa)$ on its boundary. We define $\mathbf{s}_{J}$ to be the associated $\operatorname{Spin}^{c}$ structure, and $\mathbf{s}:=\left.\mathbf{s}_{J}\right|_{X_{\kappa, n}}$. By construction, s extends the $\operatorname{Spin}^{c}$ structures induced on $\partial X_{\kappa, n}$ by $\xi_{s t}$ and $\xi_{n}^{-}(\kappa)$. This proves Part (1) of the statement. By [5] we have

$$
\frac{1}{4}\left(c_{1}\left(\left.\mathbf{s}_{J}\right|_{\widehat{X}_{\kappa, n}}\right)^{2}-3 \sigma\left(\widehat{X}_{\kappa, n}\right)-2 \chi\left(\widehat{X}_{\kappa, n}\right)\right)+1=d_{3}\left(\xi_{n}^{-}(\kappa)\right)-d_{3}\left(\xi_{s t}\right) .
$$

Figure 7b gives a natural basis $\left(\beta, x_{1}, \ldots, x_{n-1}\right)$ of $H_{2}\left(X_{\kappa, n} ; \mathbb{Z}\right)$ satisfying $\beta$. $x_{1}=1$ and $x_{i} \cdot x_{i+1}=1$ for $i=1, \ldots, n-2$. By construction and [5], the values of $c_{1}\left(\mathbf{s}_{J}\right)$ on this basis are given by $\left\langle c_{1}\left(\mathbf{s}_{J}\right), \beta\right\rangle=\operatorname{rot},\left\langle c_{1}\left(\mathbf{s}_{J}\right), x_{i}\right\rangle=\operatorname{rot}-1$, $i=1, \ldots, n-1$. We want to express the generator $[\Sigma \cup D]$ of $H_{2}\left(X_{\kappa, n} ; \mathbb{Z}\right) \cong \mathbb{Z}$ in terms of $\beta$ and $x_{1}, \ldots, x_{n-1}$. In Figure $8 \mathrm{~b}$, the classes represented by the framed circles (except the -1 -framed one corresponding to $\tau$ ) give us the new basis $\left(\beta, \beta-x_{1}, x_{1}-x_{2}, \ldots, x_{n-2}-x_{n-1}\right)$. If we define the classes $e_{1}, \ldots, e_{n-1}$ by setting

$$
e_{1}:=\beta-x_{1}, e_{i+1}-e_{i}:=x_{i}-x_{i+1}, i=1, \ldots, n-2,
$$

it is easy to check that

$$
[\Sigma \cup D]=\beta+e_{1}+\ldots, e_{n-1}
$$

and $\left\langle c_{1}\left(\mathbf{s}_{J}\right), e_{i}\right\rangle=1$ for $i=1, \ldots, n-1$. Thus,

$$
\left.\left\langle c_{1}(\mathbf{s}),[\Sigma \cup D]\right\rangle=\left\langle c_{1}\left(\mathbf{s}_{J}\right), \Sigma \cup D\right]\right\rangle=\operatorname{rot}+n-1 .
$$

This proves Part (3) of the statement. Finally, the values $\left\langle c_{1}\left(\mathbf{s}_{J}\right), e_{i}\right\rangle=1$ imply that

$$
c_{1}\left(\left.\mathbf{s}_{J}\right|_{\widehat{X}_{\kappa, n}}\right)^{2}-3 \sigma\left(\widehat{X}_{\kappa, n}\right)-2 \chi\left(\widehat{X}_{\kappa, n}\right)=c_{1}(\mathbf{s})^{2}-3 \sigma\left(X_{\kappa, n}\right)-2 \chi\left(X_{\kappa, n}\right) .
$$

Thus, Equation (5.1) implies Part (2) of the statement.

Proof of Theorem 1.4 Let $Z$ be the cobordism of Proposition 4.1 corresponding to an integer $n$. By [2, Theorem 1.2], there is a $\operatorname{Spin}^{c}$ structure $\mathbf{s}_{0}$ on the cobordism $\bar{Z}$ such that

$$
F_{\bar{Z}, \mathbf{s}_{0}}\left(c\left(\xi_{s t}\right)\right)=c\left(\xi_{n}^{-}(\kappa)\right),
$$


where $\kappa$ is the Legendrian approximation of $\tau$ described in Proposition 4.1. Since $c\left(\xi_{s t}\right) \neq 0$, we will prove that $\tilde{c}\left(\xi_{s t}, \tau, f_{S}+2 g(K)\right) \neq 0$ by showing that for an appropriate choice of $n$ the map $F_{\bar{Z}, \mathbf{s}_{0}}$ is injective.

By Proposition 4.1, the cobordism $\bar{Z}$ is exactly the cobordism induced by $-p-$ surgery along the mirror image knot $\bar{K}$, where $p=\operatorname{tb}(\kappa)+n$. Now we choose $n$ so that $p=2 g(K)$. Then, the assumption $\operatorname{sl}(\tau)=\operatorname{tb}(\kappa)-\operatorname{rot}(\kappa)=2 g(K)-1$ implies

$$
\operatorname{rot}(\kappa)+n-1=\operatorname{tb}(\kappa)-2 g(K)+1+2 g(K)-\operatorname{tb}(\kappa)-1=0 .
$$

Therefore, the $\operatorname{Spin}^{c}$ structure $\mathbf{s}$ of Proposition 5.2 satisfies $c_{1}(\mathbf{s})=0$.

By Equation (5.2) and the identification $\bar{Z}=-X_{\kappa, n}$, the Spin ${ }^{c}$ structure $\mathbf{s}_{0}$ satisfies

$$
\frac{1}{4}\left(c_{1}\left(\mathbf{s}_{0}\right)^{2}-3 \sigma\left(-X_{\kappa, n}\right)-2 \chi\left(-X_{\kappa, n}\right)\right)=-d_{3}\left(\xi_{n}^{-}(\kappa)\right)+d_{3}\left(\xi_{s t}\right)
$$

Since $\sigma\left(-X_{\kappa, n}\right)=-\sigma\left(X_{\kappa, n}\right)$, Equation (5.3) together with Proposition 5.2(2) imply $c_{1}\left(\mathbf{s}_{0}\right)^{2}=-c_{1}(\mathbf{s})^{2}=0$, therefore $c_{1}\left(\mathbf{s}_{0}\right)=0$.

Let $\mathbf{t}_{0}$ denote the restriction of $\mathbf{s}_{0}$ to $S_{-2 g(K)}^{3}(\bar{K})$. By [27, Theorem 9.19 and Remark 9.20], there is a surjective map

$$
Q: \operatorname{Spin}^{c}\left(S_{0}^{3}(\bar{K})\right) \rightarrow \operatorname{Spin}^{c}\left(S_{-2 g(K)}^{3}(\bar{K})\right)
$$

and an exact triangle

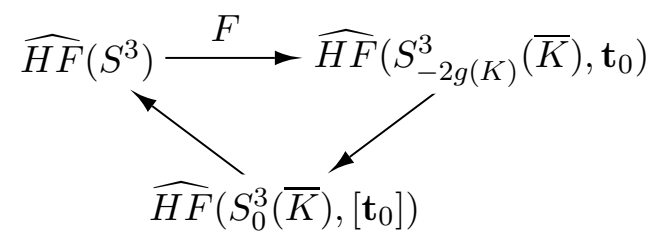

where

$$
\widehat{H F}\left(S_{0}^{3}(\bar{K}),\left[\mathbf{t}_{0}\right]\right):=\oplus_{\mathbf{t} \in Q^{-1}\left(\mathbf{t}_{0}\right)} \widehat{H F}\left(S_{0}^{3}(\bar{K}), \mathbf{t}\right) .
$$

We claim that for each $\mathbf{t} \in Q^{-1}\left(\mathbf{t}_{0}\right) \subset \operatorname{Spin}^{c}\left(S_{0}^{3}(\bar{K})\right)$ we have

$$
\left|\left\langle c_{1}(\mathbf{t}), h\right\rangle\right| \geq 2 g(K),
$$

where $h$ is a homology class generating $H_{2}\left(S_{0}^{3}(\bar{K}) ; \mathbb{Z}\right)$. In fact, since $\mathbf{s}_{0}$ extends $\mathbf{t}_{0}$, by [25, Lemma 7.10],

$$
0=\left\langle c_{1}\left(\mathbf{s}_{0}\right),[\Sigma \cup D]\right\rangle \equiv-2 g(K)+\left\langle c_{1}(\mathbf{t}), h\right\rangle \quad \bmod 4 g(K),
$$

which immediately implies the claim. 
Notice that $h$ can be represented by a genus- $g(K)$ surface (by adding the core of the 2-handle to a Seifert surface of $\bar{K})$, therefore the adjunction formula of [27, Theorem 7.1] implies $\widehat{H F}\left(S_{0}^{3}(\bar{K}),\left[\mathbf{t}_{0}\right]\right)=\{0\}$. This shows that the horizontal map $F$ of (5.4), is an isomorphism. Since $c\left(\xi_{s t}\right)$ generates $\widehat{H F}\left(S^{3}\right)$, we have $F\left(c\left(\xi_{s t}\right)\right) \neq 0$. In view of Equation (5.2), to prove that $\tilde{c}\left(\xi_{s t}, \tau, f_{S}+2 g(K)\right) \neq 0$ it suffices to show that $F=F_{\bar{Z}, \mathbf{s}_{0}}$. From the general theory we know that

$$
F=\sum_{\left\{\mathbf{s} \in \operatorname{Spin}^{c}(\bar{Z})\right.} \sum_{\left.|\mathbf{s}|_{S_{-2 g(K)}^{3}(K)}=\mathbf{t}_{0}\right\}} F_{\bar{Z}, \mathbf{s}} .
$$

Since $F$ is an isomorphism on $\widehat{H F}\left(S^{3}\right)=\mathbb{Z} / 2 \mathbb{Z}$, all the $\operatorname{Spin}^{c}$ structures contributing nontrivially the sum have the same degree shift. Moreover, if $\overline{\mathbf{s}}$ denotes the $\operatorname{Spin}^{c}$ structure conjugate to $\mathbf{s}$, by [29, Theorem 3.6] we have

$$
F_{\bar{Z}, \overline{\mathbf{s}}}\left(c\left(\xi_{s t}\right)\right)=J F_{\bar{Z}, \mathbf{s}}\left(c\left(\xi_{s t}\right)\right)
$$

where $J$ is the identification between $\widehat{H F}(Y, \mathbf{s})$ and $\widehat{H F}(Y, \overline{\mathbf{s}})$ defined in [26]. Since $J$ preserves the absolute grading, this implies that each $\mathrm{Spin}^{c}$ structure $\mathbf{s}$ with $\mathbf{s} \neq \overline{\mathbf{s}}$ contributes trivially to $F\left(c\left(\xi_{s t}\right)\right)$. Finally, from $c_{1}\left(\mathbf{s}_{0}\right)=0$ we know that $\mathbf{s}_{0}=\overline{\mathbf{s}_{0}}$, therefore by Lemma $5.1 \mathbf{s}_{0}$ is the only Spin structure on $\bar{Z}$ which extends $\mathbf{t}_{0}$. This implies $F=F_{\bar{Z}, \mathbf{s}_{0}}$ and proves $\tilde{c}\left(\xi_{s t}, \tau, f_{S}+\right.$ $2 g(K)) \neq 0$. Applying Corollary 3.9 it follows that $\tilde{c}\left(\xi_{s t}, \tau, f\right) \neq 0$ for each $f \geq f_{S}+2 g(K)$.

Proof of Theorem 1.1 Let $\tau$ be a transverse knot in the knot type $K$ with $\operatorname{sl}(\tau)=2 g(K)-1$. According to [1, Lemma 6.5] there is an open book decomposition of $S^{3}$ compatible with $\xi_{s t}$ having one binding component equal to $\tau$. Applying Theorem 1.4 the result follows for each integer surgery coefficient $n \geq 2 g(K)$. In particular, Theorem 1.4 gives a contact structure on $S_{2 g(K)}^{3}(K)$ with nonvanishing contact invariant. By the algorithm for contact surgeries described in Section 2, for $r \in \mathbb{Q}$ with $r \geq 2 g(K)$ a contact structure can be given by performing an appropriate sequence of Legendrian surgeries on the contact structure previously constructed on $S_{2 g(K)}^{3}(K)$. Since under Legendrian surgery the nonvanishing property of the contact invariant is preserved, the $3-$ manifolds $S_{r}^{3}(K)$ with $r \geq 2 g(K)$ all carry contact structures with nonvanishing contact Ozsváth-Szabó invariants. Since $c(Y, \xi) \neq 0$ implies tightness for $(Y, \xi)$, the proof of the theorem is complete. 


\section{References}

[1] K Baker, J Etnyre and J Van Horn-Morris, Cabling, contact structures and mapping class monoids, arXiv:1005.1978v 1.

[2] J Baldwin, Capping off open books and the Ozsváth-Szabó contact invariant, arXiv:0901.3797.

[3] V Chernov, Framed knots in 3-manifolds and affine self-linking numbers, J. Knot Theory Ramifications 14 (2005), 791-818.

[4] F Ding and $\mathbf{H}$ Geiges, A Legendrian surgery presentation of contact 3manifolds, Math. Proc. Cambridge Philos. Soc. 136 (2004), 583-598.

[5] F Ding, H Geiges and A Stipsicz, Surgery diagrams for contact 3-manifolds, Turkish J. Math. 28 (2004), 41-74.

[6] J Epstein, D Fuchs and M Meyer, Chekanov-Eliashberg invariants and transverse approximations of Legendrian knots, Pacific J. Math. 201 (2001), 89-106.

[7] J Etnyre, Lectures on open book decompositions and contact structures, Clay Math. Proc. 5, Proceedings of the "Floer Homology, Gauge Theory, and Low Dimensional Topology Workshop", (2006), 103-141.

[8] J Etnyre, On contact surgery, Proc. Amer. Math. Soc. 136 (2008), 3355-3362.

[9] J Etnyre and K Honda, Knots and contact geometry, I. Torus knots and the figure eight knot, J. Symplectic Geom. 1 (2001), 63-120.

[10] J Etnyre and K Honda, Cabling and transverse simplicity, Ann. of Math. (2) 162 (2005), 1305-1333.

[11] J Etnyre, D LaFountain and B Tosun, Legendrian and transverse cables of positive torus knots, arXiv:1104.0550.

[12] J Etnyre and J Van Horn-Morris, Fibered Transverse Knots and the Bennequin Bound, arXiv:0803.0758, to appear in Int. Math. Res. Not.

[13] J Etnyre and S Vela-Vick, Torsion and open book decompositions, Int. Math. Res. Not. IMRN 2010, no. 22, 4385-4398.

[14] P Ghiggini, Ozsváth-Szabó invariants and fillability of contact structures, Math. Z. 253 (2006), 159-175.

[15] P Ghiggini, K Honda and J Van Horn-Morris, The vanishing of the contact invariant in the presence of torsion, arXiv:0705.2828,

[16] K Honda, On the classification of tight contact structures. I, Geom. Topol. 4 (2000), 309-368.

[17] K Honda, W H Kazez and G Matić, Right-veering diffeomorphisms of compact surfaces with boundary, Inv. Math. 169 (2007), 427-449.

[18] J Hoste and J H Przytycki, Homotopy skein modules of orientable 3manifolds, Math. Proc. Cambridge Phils. Soc. 108 (1990), 475-488. 
[19] P Lisca and A Stipsicz, Ozsváth Szabó invariants and contact surgery, in Floer Homology, Gauge Theory, and Low-Dimensional Topology, Proceedings of the Clay Mathematics Institute 2004 Summer School, Clay Mathematics Proceedings vol. 5, A.M.S., Providence, RI.

[20] P Lisca and A Stipsicz, Ozsváth-Szabó invariants and tight contact threemanifolds, I, Geom. Topol. 8 (2004), 925-945.

[21] P Lisca and A Stipsicz, On the existence of tight contact structures on Seifert fibered 3-manifolds, Duke Math. J. 148 (2009), 175-209.

[22] P Lisca, P Ozsváth, A Stipsicz and Z Szabó, Heegaard Floer invariants of Legendrian knots in contact three-manifolds, Journal of the European Mathematical Society 11 (2009), 1307-1363.

[23] B Ozbagci, An open book decomposition compatible with rational contact surgery, Proceedings of 12th Gokova Geometry-Topology Conference 2005, International Press.

[24] P Ozsváth, Z Szabó, Knot Floer homology and the four-ball genus, Geom. Topol. 7 (2003), 615-639.

[25] P Ozsváth and Z Szabó, Absolutely graded Floer homologies and intersection forms for four-manifolds with boundary, Adv. Math. 173 (2003), 179-261.

[26] P Ozsváth and Z Szabó, Holomorphic disks and topological invariants of closed three-manifolds, Annals of Math. 159 (2004), 1027-1158.

[27] P Ozsváth and Z Szabó, Holomorphic disks and three-manifold invariants: properties and applications, Annals of Math. 159 (2004), 1159-1245.

[28] P Ozsváth and Z Szabó, Heegaard Floer homology and contact structures, Duke Math. J. 129 (2005), 39-61.

[29] Holomorphic triangles and invariants for smooth four-manifolds, Adv. Math. 202 (2006), no. 2, 326-400.

[30] P. Ozsváth, Z. Szabó and D. Thurston, Legendrian knots, transverse knots and combinatorial Floer homology, Geom. Topol. 12 (2008), 941-980.

[31] B Sahamie, Dehn Twists in Heegaard Floer Homology, Algebr. Geom. Topol. 10 (2010), 465-524.

[32] D Shea Vela-Vick, On the transverse invariant for bindings of open books, arXiv:0806.1729, to appear in J. Differential Geom.. 